\title{
Determinação comparativa da precipitação anual média na região metropolitana de
}

\section{Belém-PA}

\author{
Metropolitan region of Belém-PA Brazill a comparative determination of average annual \\ precipitation
}

Determinación comparativa de la precipitación anual promedio en la región metropolitana de Belém-PA Brasil

\begin{abstract}
Resumo
Os métodos de determinação da precipitação anual média são de grande importância para o conhecimento do regime pluviométrico de uma região, principalmente para localidades em que a precipitação é intensa e com tendência de aumento ao longo dos anos, bem como para planos de gestão dos recursos hídricos. Desta forma, o objetivo deste estudo foi determinar a precipitação anual média na Região Metropolitana de Belém (RMB), comparando e verificando as variações entre os resultados obtidos. Para tanto foram aplicados três diferentes métodos: Média Aritmética, Polígono de Thiessen e Isoietas (por meio da interpolação por Krigagem ordinária e inverso do quadrado da distância) para três condições distintas (série histórica de dados com: 37, 28 e 20 anos). Os resultados obtidos apontam que os métodos de Thiessen e das Isoietas as variações foram pouco significativas e apresentaram valores de precipitação no entorno de $2.841 \mathrm{~mm}$, ao passo de que o método de média aritmética se mostrou distante dos demais por apresentar em média 2.604 mm. Portanto, pelos resultados obtidos, conclui-se que mesmo com uma distribuição irregular das estações pluviométricas e relevo pouco acidentado os métodos de Polígono de Thiessen e de Isoietas são equivalentes para o objetivo em questão, não apresentando variação significativa entre ambos.
\end{abstract}

Palavras-chave: Precipitação anual média; Média aritmética; Polígonos de Thiessen; Isoietas.

\begin{abstract}
The knowledge of the rainfall regime has great importance for determining the average annual precipitation, mainly for locations where precipitation is intense and with an increasing tendency over the years, as well as for water resource management plans. The main goal of this research was to determine the average annual precipitation in the Metropolitan Region of Belém (MRB), by comparing and verifying the variations between the obtained results. Therefore, three different methods were applied: Arithmetic Mean, Thiessen Polygon and Isohyets (through interpolation by ordinary Kriging and inverse of the square of the distance) for three different data conditions (historical data with: 37,28 and 20 years). The obtained results, precipitation values around $2,841 \mathrm{~mm}$, shows that the variations were insignificant for Thiessen and Isohyet methods, while the Arithmetic Mean method was distant from the others with an average of 2,604 $\mathrm{mm}$. Finally, it was concluded from the obtained results that even with an irregular distribution of the rainfall stations, that is a very common situation, and a little rugged relief, the Thiessen Polygon and Isohyet methods are equivalent for the objective in question, having no significant variation between these conditions.
\end{abstract}


Keywords: Average annual precipitation; Arithmetic average; Thiessen polygons; Isohyets.

\section{Resumen}

El conocimiento del régimen de precipitaciones es de gran importancia para determinar la precipitación media anual, principalmente para lugares donde la precipitación es intensa y con tendencia creciente a lo largo de los años, así como para planes de manejo de recursos hídricos. El objetivo principal de esta pesquisa fue determinar la precipitación media anual en la Región Metropolitana de Belém (RMB), mediante la comparación y verificación de las variaciones entre los resultados obtenidos. Por tanto, se aplicaron tres métodos diferentes: Media Aritmética, Polígonos de Thiessen e Isoyetas (mediante interpolación por Kriging ordinario e inverso del cuadrado de la distancia) para tres condiciones de datos diferentes (datos históricos con: 37, 28 y 20 años). Los resultados obtenidos, valores de precipitación alrededor de $2.841 \mathrm{~mm}$, muestran que las variaciones fueron insignificantes para los métodos de Polígonos de Thiessen e Isoyetas, mientras que el método de Media Aritmética se distanció de los demás con un promedio de $2.604 \mathrm{~mm}$. Finalmente, de los resultados obtenidos, se concluyó que aún con una distribución irregular de las estaciones de lluvia, situación muy común, y un relieve poco accidentado, los métodos Polígonos de Thiessen e Isoyetas son equivalentes para el objetivo en cuestión, es decir, sin diferencias significativas variación entre ambos.

Palabras clave: Precipitación anual promedio; Media aritmética; Polígonos de Thiessen; Isoyetas.

\section{Introdução}

A chuva é um elemento natural imprescindível, visto que é responsável pela caracterização climatológica de uma determinada região. Ademais, gera informações básicas para estudos hidrológicos e ambientais, os quais, por sua vez, são essenciais para a obtenção de êxito em diversas atividades, sejam elas o planejamento de Bacias Hidrográficas (Aleixo \& Neto, 2019), o planejamento urbano e ambiental de uma região (Barboza, Caiana \& Neto, 2020) e na produção agrícola (Rossato et al., 2017).

Da mesma forma Chowdhury, Kabir, Sayed e Hossain (2016) indicam que a precipitação pluviométrica também é considerada a variável mais significativa para a identificação do aquecimento global, bem como as mudanças no estado do clima, sendo amplamente utilizada para estudar as ciências climáticas avançadas. Desta forma, conhecer a sua variação volumétrica é crucial para o adequado planejamento de políticas de mitigação e adaptação sobre as mudanças climáticas.

O estudo hidrológico da variação temporal da precipitação pluviométrica é de grande valor para qualificar os efeitos ocasionados em áreas urbanas e agrícolas, pois são inúmeros os interesses da sociedade e da engenharia nos recursos hídricos. Ocorre uma ligação entre fenômenos climáticos, escoamento superficial e projetos agrícolas e urbanos, onde o desafio não é simplesmente quantificar e qualificar o evento hidrológico, mas principalmente verificar a capacidade de prever a ocorrência de eventos extremos e suas consequências de forma mais fiel possível (Marcuzzo, 2016).

Com tamanha relevância, a compreensão da precipitação em determinada bacia hidrográfica torna-se muito ampla, já que existem diversas variáveis de tempo e espaço envolvidas na área estudada. Marciano, Barbosa e Silva (2018) indicam que a chuva é um fenômeno com elevada variabilidade espaço-temporal, já que o núcleo de chuva se modifica constantemente, tornando esta variação extremamente aleatória, de forma que a precipitação pode durar alguns minutos até várias horas ou dias, acompanhada de uma considerável amplitude de intensidade.

Diante de tal variabilidade, responsável por tornar complexa a definição do volume exato de chuva incidente numa bacia hidrográfica, Bertoni e Tucci (2002) e Collischonn e Dornelles (2015) indicam que há 3 métodos para o cálculo da chuva média (lâmina de água de altura uniforme sobre toda a área considerada, associada a um período de tempo dado), sendo eles: Método da Média Aritmética, Método de Thiessen e Método das Isoietas. Estes são bastante conhecidos e utilizados em diversos estudos hidrológicos, assim como em Al-Ozeer, Abdaki, Al-Iraqi, Al-Samman e Al-Hammadi (2020); Silva, Anjos, Gomes, Bezerra e Guimarães (2020); Lira (2019); Lee, Kim e Jun (2018); Marciano et al. (2018); Chowdhury et al. (2016); Balany (2011).

Além da questão da variabilidade espaço-temporal, outro importante fator a ser destacado é indicado nos estudos de Lee et al. (2018), os quais analisaram dois cenários de espacialização de postos pluviométricos na Coreia do Sul. O primeiro caso 
(Análise 1) compara o valor do erro de estimativa do Precipitação Média Regional (PMR) de duas bacias diferentes onde uma possui pluviômetros bem distribuídos espacialmente e a outra não. O segundo caso (Análise 2) determina o erro obtido a partir da estimativa da média pluviométrica utilizando duas distribuições de pluviômetro diferentes para a mesma bacia. Como resultado verificaram que as redes pluviométricas bem distribuídas produzem um erro menor em comparação com uma rede agrupada. Complementarmente, observaram que um erro de estimativa maior da PMR é obtido quando as estações eram espacialmente mais agrupadas do que quando há menos estações e estas estão mais dispersas. Além disso, quando a densidade de pluviômetros está abaixo de um certo nível (200 km2/estação - para o estudo em questão), o efeito da dispersão espacial das estações sobre a precisão da PMR é pequeno. Portanto, de acordo com os autores, ao estabelecer uma nova rede de medidores de chuva em uma bacia hidrográfica, tanto a densidade quanto a distribuição espacial das estações de medidor de chuva devem ser levadas em consideração.

Lundgren, Souza e Lundgren (2017) indicam que o desenvolvimento de mapas pluviométricos é a forma mais utilizada para demonstrar as previsões de chuva em uma determinada região analisada. Porém, devido o levantamento quantitativo das precipitações ser pontual, se faz necessário lançar mão das ferramentas de geoestatística, as quais auxiliam na construção de mapas de isoietas, facilitando a interpretação dos resultados obtidos. Da mesma forma, Lira (2019) enfatiza que a geoestatística é amplamente utilizada no mapeamento climático e no desenvolvimento de mapa de precipitação, porém a resposta obtida nas análises realizadas depende do tipo de interpolador utilizado.

De acordo com Ishihara et al. (2014), os métodos que mais se destacam durante a interpolação da precipitação são: polinomial global e local, inverso do quadrado da distância (IDW), interpolação usando bases de funções radiais, ou krigagem, a qual pode ser subdividida em simples, linear ordinária, universal e cokrigagem. De acordo com Medeiros, Lucio e Silva (2017), a metodologia que vem sendo mais utilizada em análises de distribuição espacial de precipitações pluviométricas em diversos locais do Brasil, é o método de krigagem.

Para a realização das análises de interpolação a fim de se obter a média de precipitação em uma determinada região, por meio do polígono de Thiessen, ou pelo método das Isoietas, pode-se utilizar os Sistemas de Informações Geográficas (SIG), os quais são ferramentas que fornecem diversas vantagens quanto ao monitoramento pluviométrico em uma ampla faixa de terra (Al-Ahmady \& Al-Jarjees, 2020; Heedan, Bapeer \& Khodakarami, 2017; Mushtaha, Van Camp \& Walraevens, 2019; Shatha \& Jafaar, 2018, Wei, Ruirui, Yan Quan, \& Jin, 2018).

A partir da utilização destes métodos é possível estimar o comportamento da precipitação para uma área onde não se verifica a presença de postos pluviométricos, oferecendo assim, subsídio para o planejamento de atividades econômicas que são diretamente ou indiretamente influenciados pela chuva (Magalhães et al., 2013).

Pelo exposto, o objetivo deste estudo foi determinar a precipitação anual média da RMB, comparando e avaliando as variações entre os resultados obtidos, através da aplicação dos métodos de Média Aritmética, Polígono de Thiessen e Isoietas (interpolação por Krigagem ordinária e IDW) para três períodos distintos (série histórica de dados com 37, 28 e 20 anos).

\section{Metodologia}

\subsection{Caracterização da área de estudo}

A RMB (Figura 1) é constituída pelos municípios de Belém, Ananindeua, Marituba, Benevides, Santa Bárbara, Santa Isabel e Castanhal. Segundo o Instituto Brasileiro de Geografia e Estatística (IBGE), a estimativa para 2020 era cerca de 2.275.032 habitantes, compondo um terço da população do estado do Pará, ocupando uma área de $3.565,8$ km². O município de Belém, que é a capital do estado, se destaca por ter a maior população com 1.499.641 habitantes e concentrar grande quantidade de áreas domiciliares (IBGE, 2010). 
Figura 1. Mapa de localização da região metropolitana de Belém.

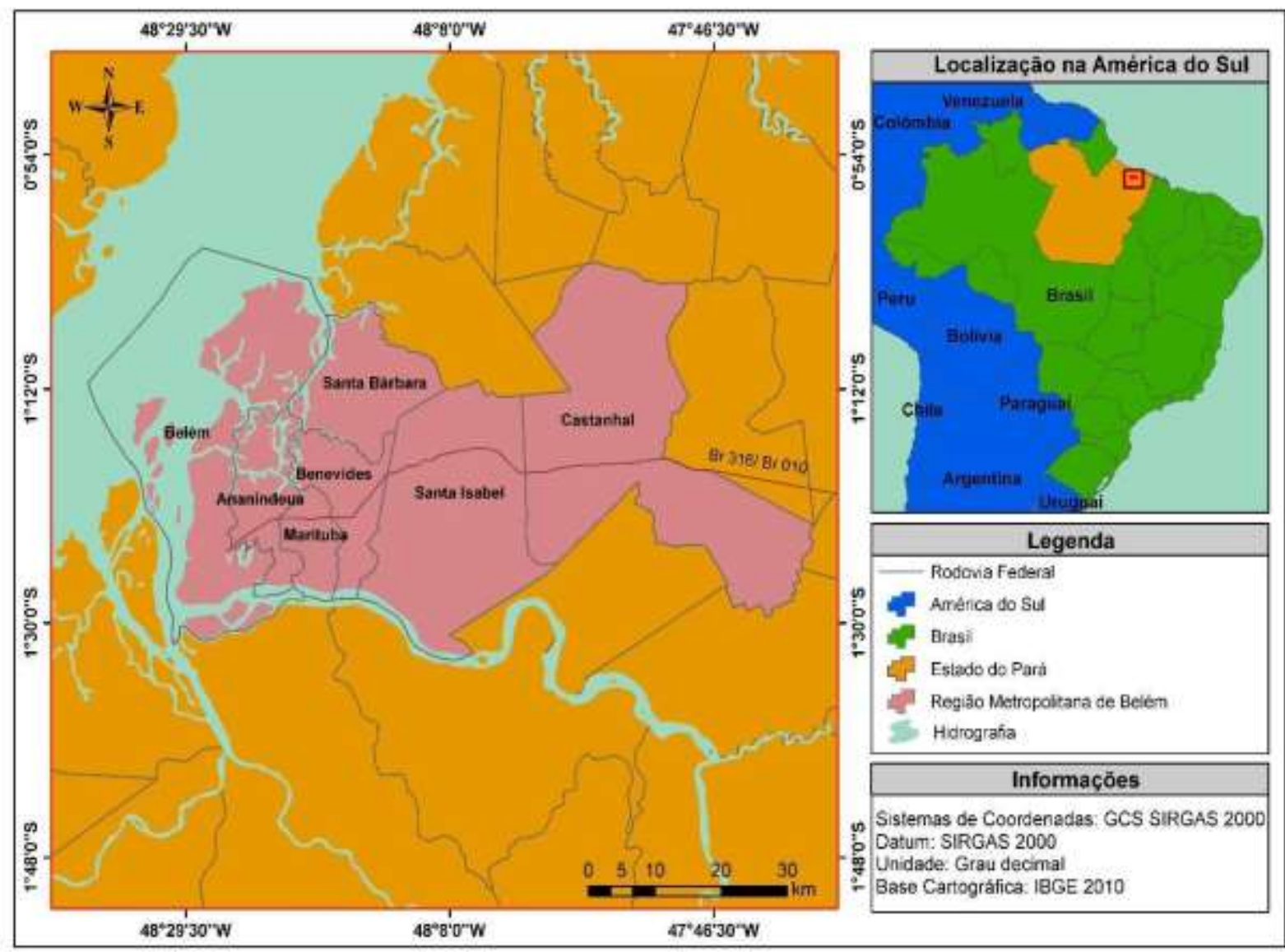

Fonte: Autores (2021).

A RMB também se caracteriza pela superfície predominantemente plana, de terras de baixa altitude e declividade suave, com hidrografia de grande porte. Os aspectos fisiográficos aliados ao histórico de falta de políticas de regulação do uso e ocupação da terra, de incentivo habitacional e reforma urbana, com altos índices de pobreza, resultaram na grande quantidade de domicílios da RMB situados em aglomerados subnormais, áreas favelizadas e/ou precárias em termos da infraestrutura urbana, da posse da terra e das condições socioeconômicas dos moradores (Ponte, Lima, Cardoso \& Rodrigues, 2013).

\subsection{Análise da precipitação anual média}

Segundo Pereira, Shitsuka, Parreira e Shitsuka (2018), o presente estudo é caracterizado pelo método quantitativo pois utilizou os dados pluviométricos de estações presentes na RMB, bem como em cidades próximas, os quais foram acessados pelo banco de dados da ANA (Agência Nacional de Águas), através da plataforma HIDROWEB. As estações disponíveis apresentam séries históricas variáveis, sendo assim, a fim de identificar alguma discrepância nos resultados, os dados foram agrupados considerando os períodos: 37, 28 e 20 anos, considerando uma quantidade de estações pluviométricas distintas: 10, 13 e 15, respectivamente.

Inicialmente, realizou-se a análise de consistência dos dados obtidos nas estações pluviométricas para conferir a homogeneidade dos dados. O método de dupla massa foi aplicado considerando a precipitação mensal acumulada do posto a consistir no eixo das ordenadas, e valores médios das precipitações mensais acumuladas das demais estações estudadas nas abscissas. Os dados são considerados homogêneos quanto mais alinhado no formato de uma reta (com coeficiente de determinação, $R^{2}$, próximos a 1), (Tucci, 2009). 


\subsubsection{Aplicação dos métodos}

Tucci (2009) informa que a determinação da altura média de precipitação em uma área é importante para o balanço hídrico de uma bacia hidrográfica. Os métodos mais usuais para determinar a precipitação média em uma área são:

\section{a) Método da Média Aritmética}

Neste método, a precipitação anual média $(\bar{h})$ será igual ao somatório das precipitações médias totais registradas em cada estação pluviométrica analisada ( $\mathrm{Pi}$ ), dividido pelo número de postos utilizados no estudo (n). Ressalta-se que este é o método mais simples de determinação da precipitação média, pois não atribui pesos aos dados amostrados. Além disso, de acordo com Mendonça, Sena e Santos (2015), os resultados só serão satisfatórios se os postos estiverem distribuídos uniformemente sobre a bacia e o terreno não deve contar com grandes variações em sua cota topográfica. Vale mencionar que tal método também é recomendado para áreas inferiores a $5.000 \mathrm{~km} 2$ que apresentem índices pluviométricos próximos. Para o cálculo deste parâmetro, utilizou-se a seguinte equação:

$$
\bar{h}=\frac{\sum P_{i}}{n}
$$

\section{b) Método dos Polígonos de Thiessen}

Antes que se aplicar a metodologia de Thiessen, primeiramente, faz-se necessário georreferenciar as estações pluviométricas trabalhadas e, desta forma, modelar os mapas contendo os resultados. Para tanto, utilizou-se o software ArcGis 10.5. Onde foi aplicada a ferramenta 3D Analyst, para realizar as espacializações de chuva na área de estudo seguindo o modelo proposto por Thiessen.

Com as estações georreferenciadas, procedeu-se o somatório do produto da precipitação de cada estação pluviométrica (Pi) pela sua respectiva área de influência $\left(A_{i}\right)$, objetivando atribuir pesos, com o intuito de aumentar o seu grau de precisão. Posteriormente, divide-se o valor obtido pela área total da região analisada $\left(A_{T}\right)$ e assim, determina-se a precipitação anual média $(\bar{h})$ para cada estação:

$$
\bar{h}=\frac{\sum\left(P_{i} * A_{i}\right)}{A_{T}}
$$

Apesar de ser considerado mais preciso que a média aritmética, o Polígono de Thiessen considera a não-uniformidade da distribuição espacial dos postos de monitoramento, o que pode torná-lo impreciso em regiões com grandes variações de relevo (Menezes, 2013).

\section{c) Método das Isoietas}

Neste método é aplicada a interpolação sobre as distâncias e a quantidade precipitada nas estações pluviométricas. Assim, são traçadas curvas de mesma precipitação, denominadas de isoietas, semelhante ao processo de traçado de curvas de nível. A média de chuva da região $(\bar{h})$ é então calculada pelo somatório da multiplicação da precipitação média das isoietas $(\bar{h})$ pelas suas respectivas áreas de influência de cada curva $\left(A_{i}\right)$, dividido pela área total $\left(A_{T}\right)$, conforme equação abaixo:

$$
\bar{h}=\frac{\sum\left(\overline{h_{l}} * A_{i}\right)}{A_{T}}
$$

Inicialmente para realizar a interpolação por krigagem ordinária, lançou-se mão da extensão Geostatistical Analyst, disponibilizada pelo ArcGis 10.5, no qual otimizou-se os dados de precipitação média das estações consideradas para a construção de semivariogramas. Elaborou-se este tipo de gráfico, com o intuito de quantificar a variação espacial na krigagem, 
onde é plotado a dispersão da semivariância (metade da variância) versus distância dos pontos amostrados. A avaliação dos dados se dará de forma interativa por meio do software ajustando o grau de dependência espacial para cada semivariograma (Silva, Rodrigues, Delgado, Oliveira, \& Sarmento, 2018; Andriotti, 2002).

Deste modo é possível verificar a dependência espacial dos gráficos, no qual foram ajustados e testados os modelos teóricos exponencial, circular, esférico e gaussiano, para identificar qual o modelo que melhor representa a distribuição de cada variável estudada, em cada período analisado, de acordo com as equações dos modelos de krigagem ordinária (Silva Pereira, Delgado \& Silva, 2016).

Em seguida, o software, automaticamente, disponibiliza parâmetros referentes aos erros obtidos por cada interpolação em relação aos dados atribuídos, como: Erro Médio (EM), Raiz Quadrada do Erro Médio (RQEM), Média Padronizada (MP), Raiz Quadrada do Erro Médio Padronizado (RQEMP) e Erro Padrão Médio (EPM); para que a partir deles se defina qual krigagem ordinária se adequa melhor ao local de análise (Mello \& Sampaio, 2019).

Adicionalmente para diversificar e comparar os resultados de interpolação de dados do estudo, adotou-se o método IDW, que é considerado um modelo mais simples que a krigagem ordinária, por atribuir pesos de acordo com o inverso da distância entre as amostras e o ponto a ser estimado, definindo, desta forma que quanto menor for a distância entre os pontos maior será o peso concedido (Righi \& Basso, 2016). No entanto, tal ferramenta não fornece o semivariograma e disponibiliza apenas o EM e a RQEM como critérios de erro para servirem de parâmetros entre os tipos de interpolação.

\section{Resultados e Discussão}

\subsection{Consistência dos dados de precipitação}

Todas as estaç̃oes avaliadas obtiveram resultados satisfatórios com relação a consistência dos dados, tomando como base a análise de dupla massa (precipitação acumulada de um posto pluviométrico em relação à média dos demais postos), comprovado pelos coeficientes de determinação $\left(R^{2}\right)$ com resultados próximos a 1, indicando boa correlação e aderência dos dados, conforme Tabela 1.

Tabela 1. Análise de consistência das estações pluviométricas.

\begin{tabular}{lcc}
\hline \multicolumn{1}{c}{ Estação Pluviométrica } & Código & Consistência \\
\hline Abaetetuba & 00148010 & $99,96 \%$ \\
Acará & 00148009 & $99,49 \%$ \\
Barcarena & 00148011 & $99,16 \%$ \\
Belém & 00148002 & $99,84 \%$ \\
Bujaru & 00148017 & $99,77 \%$ \\
Castanhal & 00147007 & $99,93 \%$ \\
Igarapé Açu & 00147010 & $99,82 \%$ \\
Mosqueiro & 00148012 & $99,96 \%$ \\
Ourém & 00147016 & $99,91 \%$ \\
Santa Cruz do Arari & 00049011 & $99,47 \%$ \\
Santa Isabel & 00148003 & $99,94 \%$ \\
Santa Maria & 00147018 & $99,49 \%$ \\
São Domingos do Capim & 00147008 & $99,98 \%$ \\
Terra Alta & 00147017 & $99,92 \%$ \\
Vigia & 00048006 & $99,96 \%$ \\
\hline
\end{tabular}

Fonte: Autores (2021). 
Como exemplo, Figura 2, a precipitação acumulada da estação de Abaetetuba (eixo das ordenadas) pela média acumulada das demais estações pluviométricas (eixo das abcissas). Neste caso foi obtido um $\mathrm{R}^{2}$ igual a 0,9996 apontando para um comportamento semelhante a uma reta.

Figura 2. Análise de Consistência do posto de Abaetetuba.

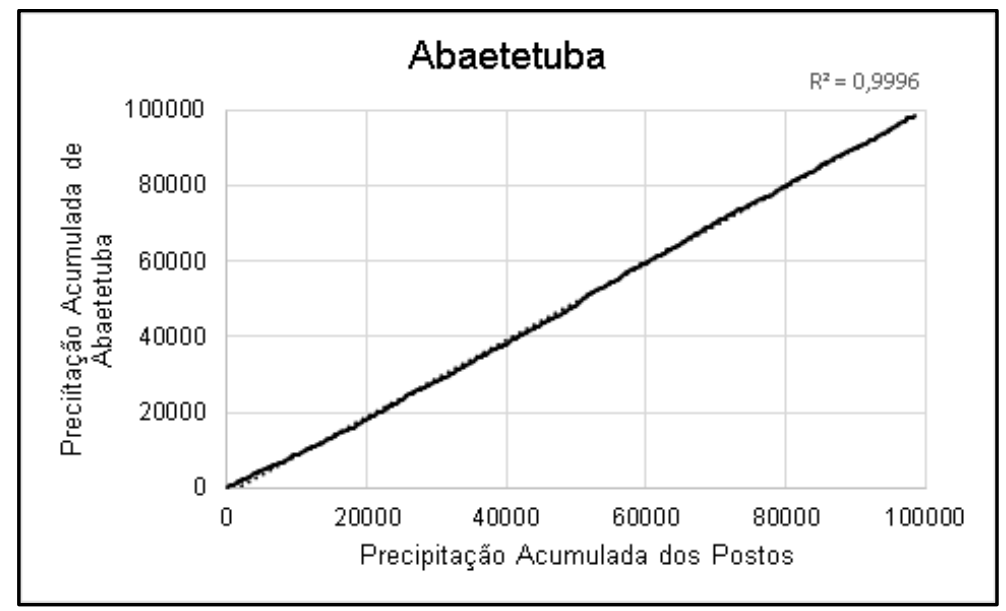

Fonte: Autores (2021).

A conferência da homogeneização dos dados anuais de precipitação é importante para identificar se há desproporcionalidade ou mudança de inclinação na distribuição dos pontos no gráfico, indicando possíveis anormalidades na estação, assim como mudança de local, avarias no aparelho, e até alterações no método de observação (Mendes \& Zukowski, 2017)

\subsection{Média Aritmética}

Para a aplicação deste método, inicialmente, determinou-se a precipitação anual média de cada estação pluviométrica estudada, assim como exposto na Tabela 2.

Tabela 2. Precipitação anual média dos postos pluviométricos avaliados.

\begin{tabular}{lc}
\hline \multicolumn{1}{c}{ Estação Pluviométrica } & $\begin{array}{c}\text { Precipitação Anual } \\
\text { Média (mm) }\end{array}$ \\
\hline Abaetetuba & $2.591,88$ \\
Acará & $2.710,85$ \\
Barcarena & $2.179,45$ \\
Belém & $3.010,14$ \\
Bujaru & $2.401,55$ \\
Castanhal & $2.600,03$ \\
Igarapé Açu & $2.403,59$ \\
Mosqueiro & $3.080,90$ \\
Ourém & $2.180,94$ \\
Santa Cruz do Arari & $2.506,96$ \\
Santa Isabel & $2.942,38$ \\
Santa Maria & $2.243,80$ \\
São Domingos do Capim & $2.273,09$ \\
Terra Alta & $2.674,96$ \\
Vigia & $2.753,89$ \\
\hline
\end{tabular}

Fonte: Autores (2021). 
Ressalta-se que foram utilizados todos os resultados de precipitação para determinar a precipitação média referente ao período de 20 anos. No entanto, as estações de Bujaru e Santa Cruz do Arari não apresentam dados históricos suficientes para serem aplicados no intervalo de 28 anos. Já para 37 anos, além de não contar com os postos anteriormente citados, também não consideraram as estações localizadas nos municípios de Terra Alta, Santa Maria e Ourém.

Em seguida, determinou-se para os intervalos de tempo de 37, 28 e 20 anos, as médias aritméticas de precipitação anual para a RMB, obtendo-se, dessa forma, $2.655 \mathrm{~mm}, 2.588 \mathrm{~mm}$ e $2.570 \mathrm{~mm}$, respectivamente.

De acordo com Borges et al. (2019), durante um período de 16 anos, a pluviosidade da cidade de Belém apresentou precipitação uniforme superior a $3.000 \mathrm{~mm}$ por ano, o que confirma essa alta pluviosidade no decorrer dos anos, Tabela 2. Dados semelhantes foram obtidos por Albuquerque, Souza, Oliveira e Junior (2010) durante o período de 1978 a 2008, onde, afirma-se que os maiores índices estão concentrados no nordeste paraense, com precipitação superior a $2.000 \mathrm{~mm}$, englobando as mesorregiões do Marajó, Metropolitana de Belém e Nordeste Paraense. Destaca-se que a mesorregião Metropolitana de Belém é a que apresenta o maior índice pluviométrico do Estado do Pará.

Na Figura 3, é indicada a localização das 15 estações pluviométricas consideradas nesta pesquisa e o valor da média aritmética da precipitação para o período de 20 anos. Além disso, para os demais intervalos os resultados são semelhantes, pois além do valor da precipitação média calculada, a única diferença entre os mapas fica a cargo da quantidade de estações pluviométricas adotadas em cada intervalo de tempo.

Figura 3. Mapa de localização das estações pluviométricas consideradas na pesquisa.

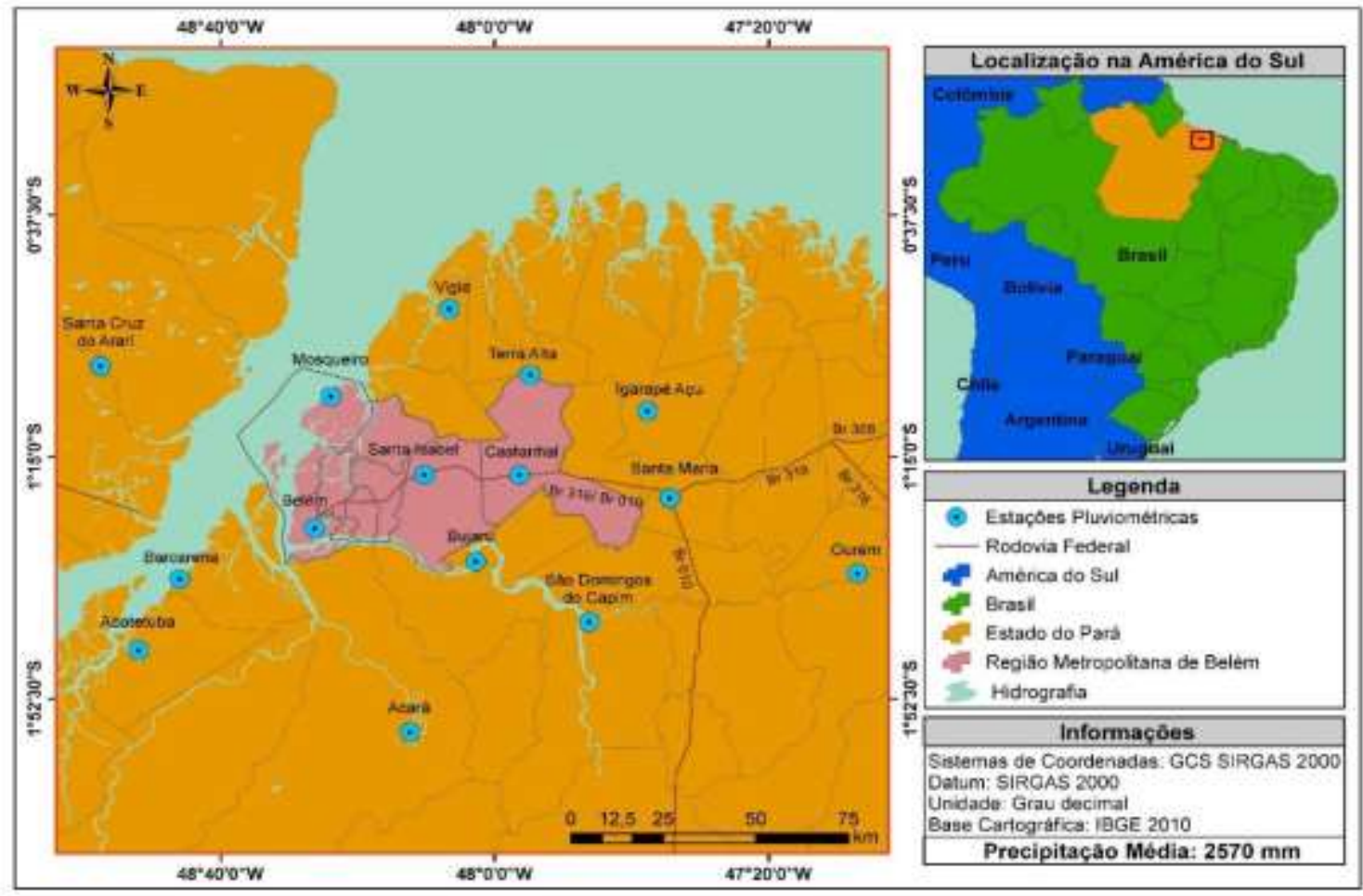

Fonte: Autores (2021).

\subsection{Polígono de Thiessen}

Para os intervalos de tempo considerados (37, 28 e 20 anos), as precipitações anuais médias, segundo a metodologia dos Polígonos de Thiessen são, respectivamente de $2.870 \mathrm{~mm}, 2.865 \mathrm{~mm}$ e $2.836 \mathrm{~mm}$. Nota-se que, apesar dos períodos apresentarem áreas de influências diferentes para cada posto pluviométrico (já que para cada período analisado há um número diferente de 
estações pluviométricas), os resultados demonstram proximidade entre os valores. Destaca-se que, em termos de comportamento climático regional, na Região Amazônica o sistema mais atuante é a ZCIT (Zona de Convergência Intertropical). Tal fato intensifica o regime de chuva na região, especialmente em períodos mais chuvosos (Gonçalves, Araújo, Lima \& Rocha, 2013)

Na Figura 4 pode ser observada a espacialização dos resultados, com a precipitação média referente a cada polígono e a área de influência específica dos postos pluviométricos para o período de 20 anos. Nota-se que os maiores índices pluviométricos se encontram próximos das estações localizadas a leste da área de estudo e os polígonos apresentam uma variação de pluviosidade de 2.244 a $3.081 \mathrm{~mm}$, sendo que para este cálculo, o software utilizou apenas as estações de Belém, Mosqueiro, Santa Isabel, Bujarú, São Domingos do Capim, Castanhal e Terra Alta, seguindo a metodologia do presente modelo.

Figura 4. Polígono de Thiessen considerando-se as estações pluviométricas de 20 anos de observação.

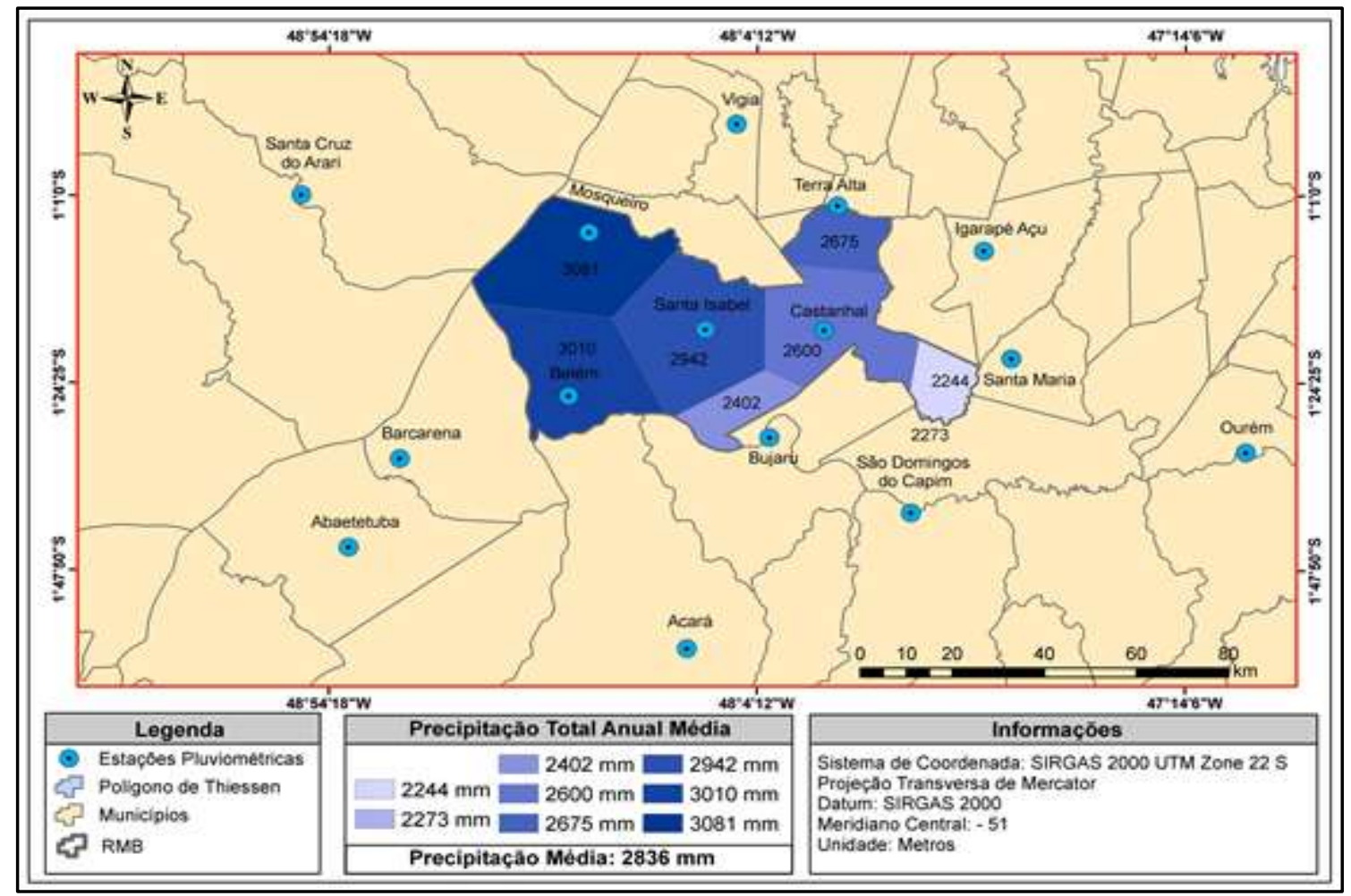

Fonte: Autores (2021).

\subsection{ISOIETAS}

A partir dos parâmetros estatísticos analisados e por meio do ajuste aos semivariogramas dentre os tipos de krigagens ordinária dos períodos de 37, 28 e 20 anos, destacam-se respectivamente: exponencial, gaussiano e circular. Para representar o estudo, optou-se pelo período de 37 anos (apresentado pelo modelo exponenial), como pode ser observado na Figura 5, onde os dados pluviométricos acompanham o comportamento da curva do modelo, semelhante ao estudo de Farias, Francisco e Senna (2017) que realizaram o ajuste do semivariograma para 18 séries pluviométricas obtendo um comportamento moderadamente não estacionário, ou seja, a medida que a distância entre as amostras aumenta, a variância tende a apresentar valores menores. 
Figura 5. Semivariograma exponencial para o período de 37 anos.

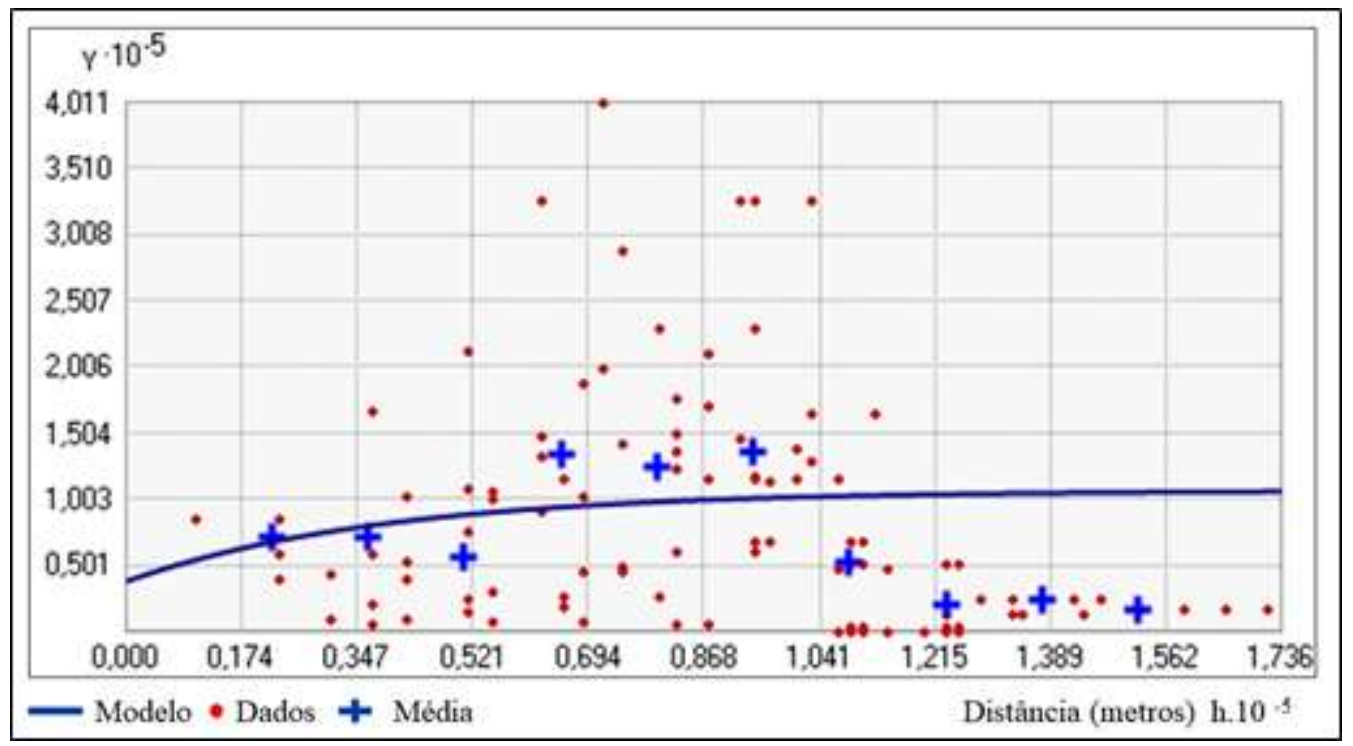

Fonte: Autores (2021).

O cálculo das isoietas foi determinado por diferentes interpolações, os quais se mostraram mais adequados para serem utilizados, após realização de análise dos parâmetros de erros apresentados na Tabela 3. Tal fato assemelha-se ao obtido no estudo de Uliana, Reis, Silva e Xavier (2013), no qual, a partir dos testes de semivariância, verificou-se que os melhores resultados foram alcançados para os modelos esférico, gaussiano e exponencial, representando mais adequadamente a precipitação mensal e anual para o Estado do Espírito Santo.

Para a análise dos erros, o modelo exponencial obteve o menor valor para o período de 37 anos (Tabela 3), assim como fora observado em alguns estudos, tal qual Wanderley, Amorim e Carvalho (2012) que aplicaram o mesmo modelo exponencial na região de Alagoas para um período não chuvoso, e Mello e Sampaio (2019) que tiveram resultados positivos para o período chuvoso no Estado do Paraná. Porém, apesar dos bons resultados em estudos anteriores, pode-se notar, na Tabela 3, que os modelos que mais se destacam nos períodos de 28 e 20 anos, respectivamente, são o gaussiano e circular em comparação a média de precipitação da RMB. 
Tabela 3. Parâmetros obtidos por meio da interpolação utilizando Krigagem Ordinária.

\begin{tabular}{|c|c|c|c|c|c|c|c|}
\hline $\begin{array}{c}\text { Período } \\
\text { Mínimo de } \\
\text { Precipitação }\end{array}$ & $\begin{array}{c}\text { Modelo } \\
\text { Selecionado }\end{array}$ & $\begin{array}{l}\text { Erro } \\
\text { Médio } \\
\text { (EM) }\end{array}$ & $\begin{array}{c}\text { Média } \\
\text { Ponderada } \\
\text { (MP) }\end{array}$ & $\begin{array}{c}\text { Raiz } \\
\text { Quadrada do } \\
\text { Erro Médio } \\
\text { Padronizado } \\
\text { (RQEMP) }\end{array}$ & $\begin{array}{c}\text { Raiz } \\
\text { Quadrada } \\
\text { do Erro } \\
\text { Médio } \\
\text { (RQEM) }\end{array}$ & $\begin{array}{c}\text { Erro } \\
\text { Padrão } \\
\text { Médio } \\
\text { (EPM) }\end{array}$ & $\begin{array}{l}\text { Precipitação } \\
\text { Anual Média }\end{array}$ \\
\hline $\begin{array}{c}\text { Valor ideal } \\
\text { (Mello \& } \\
\text { Sampaio, } \\
\text { 2019) }\end{array}$ & - & $\begin{array}{l}\text { Próx. } \\
\text { de } 0\end{array}$ & Próx. de 0 & Próx. de 1 & Próx. de 0 & $\begin{array}{c}\text { Próx. do } \\
\text { RQEM }\end{array}$ & - \\
\hline \multirow{4}{*}{37 anos } & Circular & 20,505 & 0,053 & 0,994 & 318,459 & 321,979 & $2.701 \mathrm{~mm}$ \\
\hline & Esférico & 18,227 & 0,046 & 0,943 & 293,749 & 315,007 & $2.745 \mathrm{~mm}$ \\
\hline & Exponencial & 15,166 & 0,038 & 0,937 & 291,998 & 315,308 & $2.768 \mathrm{~mm}$ \\
\hline & Gaussiano & 19,599 & 0,049 & 0,971 & 308,999 & 320,075 & $2.709 \mathrm{~mm}$ \\
\hline \multirow{4}{*}{28 anos } & Circular & 5,679 & 0,029 & 0,878 & 217,422 & 269,611 & $2.833 \mathrm{~mm}$ \\
\hline & Esférico & 5,488 & 0,026 & 0,820 & 217,815 & 282,352 & $2.835 \mathrm{~mm}$ \\
\hline & Exponencial & 8,323 & 0,026 & 0,791 & 229,368 & 297,244 & $2.818 \mathrm{~mm}$ \\
\hline & Gaussiano & $-1,250$ & $\mathbf{0 , 0 0 8}$ & 1,028 & 225,546 & 248,863 & $2.842 \mathrm{~mm}$ \\
\hline \multirow{4}{*}{20 anos } & Circular & 4,740 & 0,022 & 0,922 & 222,730 & 265,285 & $2.810 \mathrm{~mm}$ \\
\hline & Esférico & 8,345 & 0,033 & 0,909 & 221,942 & 264,193 & $2.811 \mathrm{~mm}$ \\
\hline & Exponencial & 6,690 & 0,022 & 0,845 & 223,554 & 276,261 & $2.799 \mathrm{~mm}$ \\
\hline & Gaussiano & 8,587 & 0,030 & 0,893 & 221,574 & 265,348 & $2.780 \mathrm{~mm}$ \\
\hline
\end{tabular}

Fonte: Autores (2021).

Constata-se, pela Tabela 4, que os modelos de krigagem ordinária apresentaram erros consideravelmente menores quando comparados ao método IDW, para os períodos de 28 e 20 anos, desta forma, os dois modelos, gaussiano e circular, foram utilizados para realizar a espacialização das chuvas no mapa de precipitações médias. Furtado, Pereira e Castro (2017), realizaram semelhante estudo e indicaram a krigagem como o método de interpolação mais adequado para estimar precipitação pluviométrica do nordeste paraense e RMB.

Tabela 4. Comparativo entre os resultados dos modelos de krigagem e IDW.

\begin{tabular}{ccccc}
\hline $\begin{array}{c}\text { Período Mínimo de } \\
\text { Precipitação }\end{array}$ & Modelo de Interpolação & $\begin{array}{c}\text { Erro Médio } \\
\text { (EM) }\end{array}$ & $\begin{array}{c}\text { Raiz Quadrada do } \\
\text { Erro Médio (RQEM) }\end{array}$ & $\begin{array}{c}\text { Precipitação } \\
\text { Anual Média }\end{array}$ \\
\hline $\begin{array}{c}\text { Valor ideal (Mello \& } \\
\text { Sampaio, 2019) }\end{array}$ & - & Próx. de 0 & Próx. de 0 & - \\
\hline \multirow{2}{*}{37 Anos } & Krigagem Exponencial & 15,166 & 291,998 & $2768 \mathrm{~mm}$ \\
\hline \multirow{2}{*}{28 Anos } & IDW & 29,407 & 291,389 & $2825 \mathrm{~mm}$ \\
\hline \multirow{2}{*}{20 Anos } & Krigagem Gaussiana & $-1,250$ & 225,546 & $2842 \mathrm{~mm}$ \\
& IDW & 27,302 & 260,087 & $2813 \mathrm{~mm}$ \\
\hline
\end{tabular}

Fonte: Autores (2021).

Por outro lado, ao avaliar-se o período de 37 anos de série histórica, constata-se que não houve uma diferença significativa entre os resultados dos dois métodos (Tabela 4). Isto porque, apesar de a krigagem exponencial apresentar o menor EM, a interpolação IDW obteve o menor RQEM, alinhando-se melhor as médias de precipitação dos outros períodos, assim como foi realizado nos estudos de Magalhães et al. (2013), no qual o modelo de interpolação IDW, obteve menores índices de 
erro para a espacialização da chuva na região norte do estado do Espírito Santo em comparação com a krigagem ordinária. Desta forma, optou-se por utilizar a interpolação IDW para espacialização desse período.

Na Figura 6, observa-se que, apesar dos valores da precipitação anual média serem próximos, cada período apresenta as suas especificidades em relação à espacialização das chuvas. Isto torna-se mais evidenciado ao se comparar diferentes tipos de interpolação, pois a krigagem ordinária apresenta isoietas mais suavizadas. Tal como foi encontrado por Paiva (2008) onde os campos de precipitação obtidos pela krigagem ordinária são mais suaves.

Figura 6. Espacialização por Krigagem Gaussiana para o período de 28 anos (A); Espacialização por Krigagem Circular para o período de 20 anos (B).

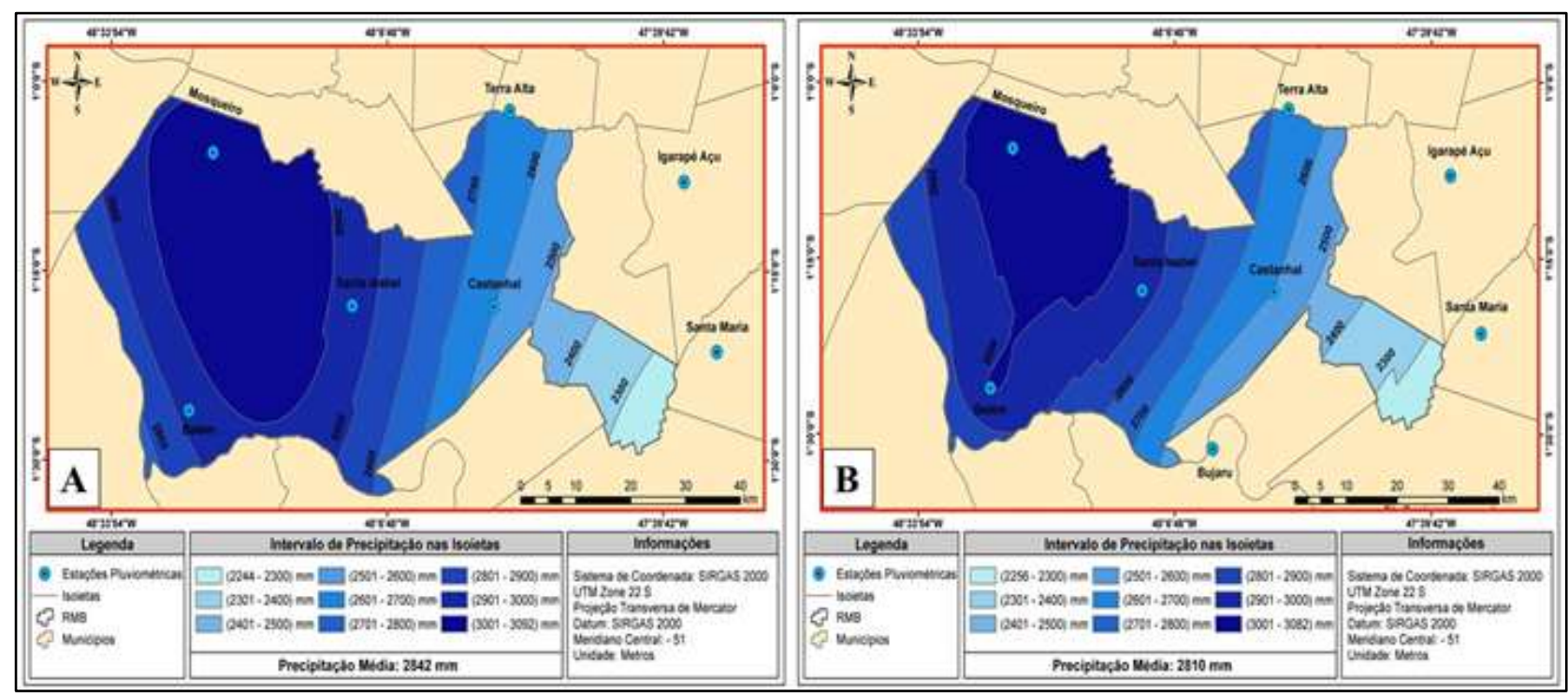

Fonte: Autores (2021).

Em contrapartida, o método IDW expõe um modelo arredondado ao redor das estações semelhante aos estudos de Righi e Basso (2016), no qual avaliou métodos de interpolação de chuva e obteve resultado de contornos arredondados nos pontos de amostragem, isto é demonstrado pela condição estatística do método, pois cada ponto de amostragem tende a formar um raio semelhante em todas as direções que possibilita na formação de círculos. 
Figura 7. Espacialização por IDW para o período de 37 anos.

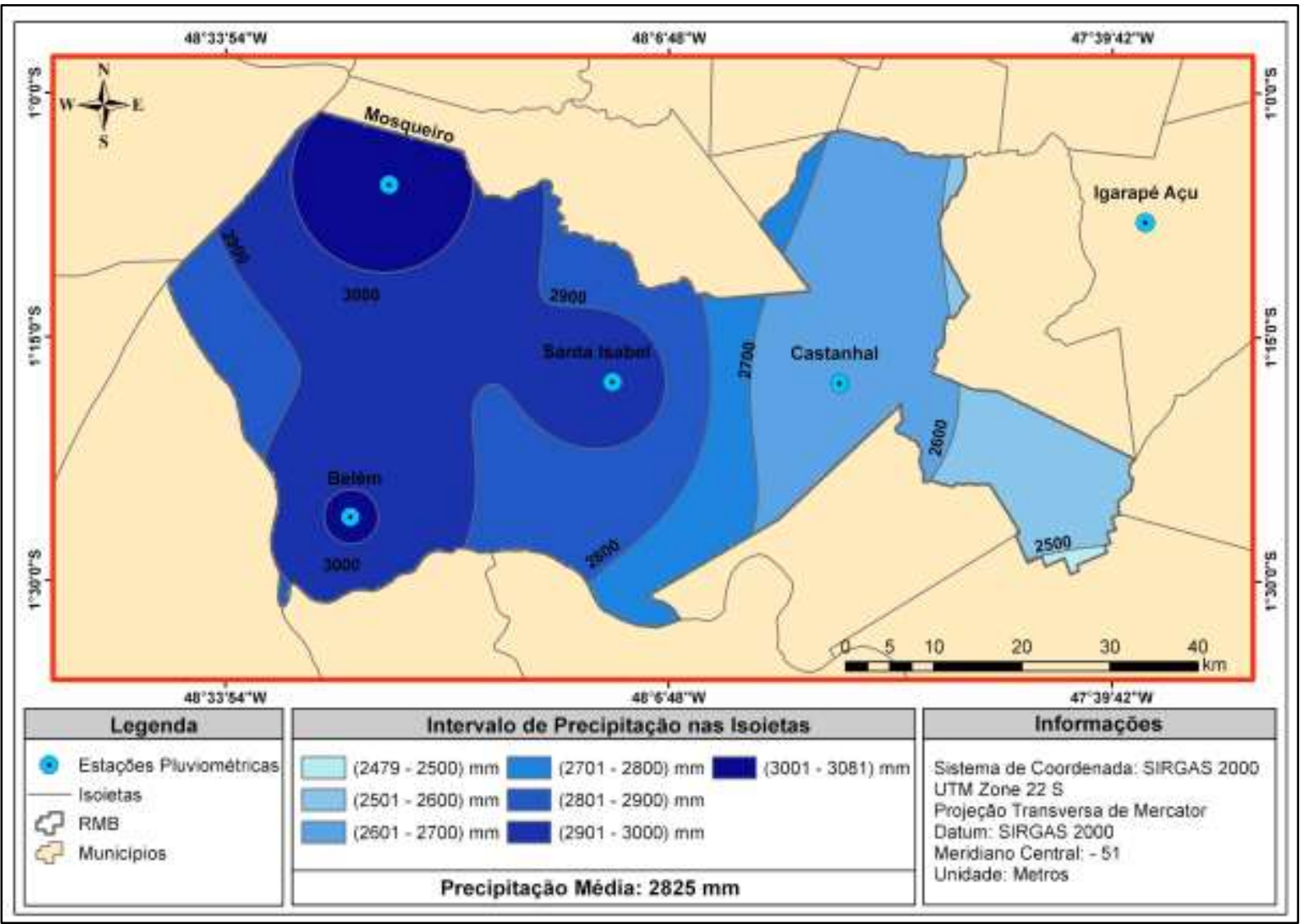

Fonte: Autores (2021).

Os resumos dos resultados pelos três métodos são arrolados na Tabela 5, sendo descrito o método de determinação de precipitação média, o período de observação dos dados e a precipitação anual média encontrada.

Tabela 5. Precipitações médias anuais por método analisado em diferentes períodos.

\begin{tabular}{ccc}
\hline Método & Período Mínimo & Precipitação Anual Média \\
\hline \multirow{3}{*}{ Média Aritmética } & 37 anos & $2.655 \mathrm{~mm}$ \\
& 28 anos & $2.588 \mathrm{~mm}$ \\
& 20 anos & $2.570 \mathrm{~mm}$ \\
\hline \multirow{2}{*}{ Pol. De Thiessen } & 37 anos & $2.870 \mathrm{~mm}$ \\
& 28 anos & $2.865 \mathrm{~mm}$ \\
& 20 anos & $2.836 \mathrm{~mm}$ \\
\hline \multirow{2}{*}{ Isoietas } & 37 anos & $2.825 \mathrm{~mm}$ \\
& 28 anos & $2.842 \mathrm{~mm}$ \\
& 20 anos & $2.810 \mathrm{~mm}$ \\
\hline
\end{tabular}

Fonte: Autores (2021).

De acordo com estudos realizados pela Agência Nacional de Águas (2017) envolvendo estações nos municípios da RMB onde os totais médios anuais foram distribuídos através da interpolação, pelo método da krigagem, a chuva total anual média para essa área foi estimada em $2.944 \mathrm{~mm}$. A precipitação média calculada por Thiessen e isoietas foi de $2841 \mathrm{~mm}$, nesse sentido, nota-se uma variação de aproximadamente $100 \mathrm{~mm}$ ao se comparar ambos os resultados. 
Assim também, apesar de utilizar apenas as séries históricas do município de Belém, Lira, Lopes, Chaves, Santana e Fernandes (2020) obtiveram média anual de $3.070 \mathrm{~mm}$ e Campos, Mota e Santos (2015) resultaram em médias variando entre $2.500 \mathrm{~mm}$ a $3.900 \mathrm{~mm}$, avaliando um período de eventos extremos, que podem confirmar que as estimativas de precipitação pelos métodos de Thiessen e isoieta resultaram em valores próximos considerando os demais municípios da RMB.

Ainda com relação a Tabela 5 evidencia-se que as precipitações médias anuais obtidas pelos polígonos de Thiessen e isoietas apresentam resultados similares e pouco variáveis, com pequenas variações de no máximo $60 \mathrm{~mm}$, sugerindo que ambos os métodos são mais adequados quando comparados com o primeiro. Tal fato pode estar relacionado à característica do relevo da RMB, a qual possui uma superfície plana na maior parte de sua área. Tal propriedade influencia positivamente nos valores de média obtidos pelo método de Thiessen, já que como é indicado por Marciano et al. (2018) e Menezes (2013), este método não é satisfatório para aplicação em terrenos acidentados.

Por outro lado, os resultados referentes ao método de média aritmética se apresentaram menores do que os outros dois, o que pode estar relacionado, principalmente, à simplicidade do método, o qual não considera a aplicação de pesos ou variações espaciais para a obtenção dos resultados.

\section{Conclusão}

O presente trabalho tratou da determinação da precipitação média na Região Metropolitana de Belém por três diferentes métodos. Com os resultados obtidos neste estudo conclui-se que os melhores resultados estão associados as precipitações obtidas pelos métodos Thiessen e Isoietas, principalmente pelo fato de a RMB possuir um relevo plano. Vale mencionar, os períodos das séries históricas utilizados e consequentemente a quantidade de estações também influenciaram nos resultados, porém com menor significância.

Dentre os métodos utilizados para a determinação da precipitação anual média na RMB, a média aritmética e polígonos de Thiessen são aplicados de forma mais simplificada quando comparados ao método de Isoietas, já que este último necessita de um ajuste mais detalhado para sua determinação, sendo necessária a verificação de erros estatísticos (por krigagem ou IDW). Embora o IDW não seja o modelo mais indicado para a aplicação no método de isoietas, como é a krigagem, também apresentou resultados satisfatórios para os valores de chuva anual média.

Sendo assim, após a aplicação dos três métodos e suas respectivas análises, pode-se afirmar que o Polígono de Thiessen e Isoietas apresentaram resultados de precipitação anual média na ordem de $2.841 \mathrm{~mm}$, indicados como mais adequados e com menores variações, quando comparados com o método de média aritmética que apresentou resultados em torno de 2.604 $\mathrm{mm}$. Isto se deve aos dois primeiros métodos considerarem a área de estudo e atribuição de pesos para o cálculo, diferentemente do método da Média Aritmética que utiliza a média direta.

Por fim, destaca-se que os métodos de determinação da precipitação anual média são de grande importância para o conhecimento do regime pluviométrico de uma região, principalmente para localidades em que a precipitação é intensa e tende a aumentar ao longo dos anos. Desta forma, estudos que objetivem alcançar tais informações pluviométricas podem ser de grande valia para auxiliar o poder público na tomada de decisões quanto ao gerenciamento de recursos hídricos e de maneira a prevenir problemas urbanos, prejuízos econômicos e sociais.

Finalmente, não é possível afirmar que como regra geral que um método é superior ao outro sem um estudo comparativo e neste caso recomenda-se sempre que necessário a comparação entre os métodos para melhor conclusão.

Acredita-se que o presente trabalho, futuramente, auxilie o planejamento hídrico da Região Metropolitana Belém, como também possa respaldar novos trabalhos a serem desenvolvidos. Posteriormente, sugere-se a ampliação da pesquisa para as demais regiões do estado do Pará, com o intuito de gerar mais informações que beneficiem a região norte do Brasil. 


\section{Referências}

Agência Nacional de Águas (2017). Estudos Hidrogeológicos para a Definição de Estratégias de Gestão das Águas Subterrâneas da Cidade de Belém/PA e Municípios Adjacentes: relatório parcial. p. 41.

Albuquerque, M. F., Souza, E. B., Oliveira, M. C. F. \& Junior, J. A. S. (2010). Precipitação nas mesorregiões do estado do Pará: climatologia, variabilidade e tendências nas últimas décadas (1978-2008). Revista Brasileira de Climatologia. 6.

Al-Ahmady, K. K., \& Al-Jarjees, S. D. (2020). The usage of gis for the devastated urban centers management and preservation of monuments study case: NabiJarjis district In Mosul City In Iraq. Iraqi Geological Journal, 53, 57-69.

Aleixo, N. C. R., \& Neto, J. C. A. S. (2019). Caracterização da precipitação pluviométrica do médio Solimões - AM. Acta Geográfica, 13(31), 104-120.

Al-Ozeer, A., Abdaki, M. A., Al-Iraqi, A. R., Al-Samman, S. H., \& Al-HAmmadi, N. A. (2020). Estimation of mean areal rainfall and missing data by using gis in nineveh, Northern Iraq. Iraqi Geological Journal, 53(1), 93-103.

Andriotti, J. L. S. (2002). Notas de geoestatística. Revista Acta Geologica Leopoldensia. 35(55): 3-14.

Balany, F. (2011). Different ways of calculating catchment rainfall: case in Indonesia. Civil Engineering Forum, 20(1), 1175-1182.

Barboza, E. N., Caiana, C. R. A., \& Neto, F. D. C. B. (2020). Análise da precipitação pluviométrica na Região do Centro-Sul Cearense: Um estudo do período (1980-2009). Research, Society and Development, 9(6).

Bertoni, J. C., \& Tucci, C. E. M. (2002). Precipitação. In C. E. M. Tucci (Org.), Hidrologia: Ciência e Aplicação (cap. 5). ABRH.

Borges, A. E. F., Souza, A. M. B., Monteiro, A. D. S., Silva, B. M., Soares, C. D. A., Silva, D. A. C., \& Lobato, W. T. D. S. (2019). Comparação da Precipitação Pluviométrica de 12 Cidades Paraenses. As ciências exatas e da terra no século XXI [recurso eletrônico]: volume 2 / Organizadores Júlio César Ribeiro, Carlos Antônio dos Santos. Ponta Grossa, PR: Atena Editora. 1-388. Recuperado de https://www.atenaeditora.com.br/post-artigo/23275.

Campos, T. L. O. B., Mota, M. A. S., \& Santos, S. R. Q. (2015). Eventos extremos de precipitação em Belém-PA: uma revisão de notícias históricas de jornais. Revista Ambiente \& Água, 10(1), 182-194.

Chowdhury, M. A. I., Kabir, M. M., Sayed, A. F., \& Hossain, S. (2016). Estimation of rainfall patterns in Bangladesh using different computational methods (arithmetic average, thiessen polygon and isohyet). Journal of Biodiversity and Environmental Sciences, 8(1), 43-51.

Collischonn, W., \& Dornelles, F. (2015). Hidrologia para engenharia e ciências ambientais (2 ed.). ABRH.

Farias, O. G., Francisco, C. N., \& Senna, M. C. A. (2017). Avaliação de métodos de interpolação espacial aplicados à pluviosidade em região montanhoso no litoral sul do estado Rio de Janeiro. Revista Brasileira de Climatologia, 21.

Furtado, L., Pereira, C., \& Castro, A. D. C. (2017). Análise de métodos de interpolação para a espacialização da precipitação pluviométrica da região metropolitana de Belém e Nordeste paraense. In Embrapa Amazônia Oriental-Artigo em anais de congresso (ALICE). Anais do Congresso Brasileiro de Cartografia.

Gonçalves, L., Araújo, S., Lima, A., \& Rocha, E. (2013). Estimativa da distribuição da precipitação pluviométrica e de vazões mínimas de referência para as bacias dos rios branco e negro. Anais do Simpósio Brasileiro de Recursos Hídricos. https://abrh.s3.sa-east1.amazonaws.com/Sumarios/155/6fb75cde593139d234a3cc4f27aeeaaa_d99a5c0feaac3b6ca1f45bff9 653e7d4.pdf .

Heedan, M. O., Bapeer, G. B., \& Khodakarami, L. (2017). Estimation the volume of runoff using natural resources conservation service method and geographic information system in Koya Basin, Sulaimaniya, Iraq. Iraqi Geological Journal, 50, 100-120.

Instituto Brasileiro de Geografia e Estatística - IBGE. Recuperado de https://cidades.ibge.gov.br/brasil/pa/belem/panorama.

Ishihara, J. H., Fernandes, L.L., Duarte, A. A. M., Duarte, A. R. C. L. M., Ponte, M. X., \& Loureiro, G. E. (2014). Quantitative and spatial assessment of precipitation in the Brazilian Amazon (Legal Amazon) - (1978 to 2007). Revista Brasileira de Recursos Hídricos, 19 (1), $29-39$.

Lee, J., Kim, S., \& Jun, H. (2018). A study of the influence of the spatial distribution of rain gauge networks on areal average rainfall calculation. Water, 10.

Lira, B. R. P. Avaliação do Comportamento e da Tendência Pluviométrica na Amazônia Legal no Período de 1986 a 2015. (2019). (Dissertação de Mestrado). Programa de Pós Graduação em Engenharia Civil, Universidade Federal do Pará - UFPA, Belém, PA, Brasil.

Lira, B. R. P., Lopes, L. N. A, Chaves, J. R., Santana, L. R., \& Fernandes, L. L. (2020). Identificação de Homogeneidade, Tendência e Magnitude da Precipitação em Belém (Pará) entre 1968 e 2018. Anuário do Instituto de Geociências, 43(4), 426-439.

Loitzenbauer, E., \& Adam, K. (2011). Análise espacial da precipitação média mensal na Sub-Bacia do Taboão (RS), durante os anos de 2001 a 2008. Anais do Simpósio Brasileiro de Recursos Hídricos. https://www.abrhidro.org.br/SGCv3/publi cacao.php?PUB=3\&ID=81\&PUBLICACAO=SIMPOSIOS

Lundgren, W. J. C., Souza, I. F., \& Lundgren, G. A. (2017). Krigagem na construção de mapa pluviométrico do Estado de Sergipe. Revista Brasileira de Geografia Física, 10(1), 13-22.

Magalhães, I. A. L., Almeida, K. L., Thiago, C., Junior, B., Zanetti, S. S., \& Cecílio, R. (2013). Análise de métodos de interpolação para espacialização da precipitação pluvial na região Norte do estado do Espírito Santo, Brasil. Anais do Simpósio Brasileiro de Sensoriamento Remoto-SBSR. Foz do Iguaçu, PR, Brasil, 16.

Marciano, A. G., Barbosa, A. A., \& Silva, A. P. M. (2018). Cálculo de precipitação média utilizando método de Thiessen e as linhas de cumeada. Revista Ambiente \& Água, 13(1). 
Marcuzzo, F. F. N. (2016). A distribuição espacial da chuva mensal e anual no território do município de São Paulo. Anais do Encontro Técnico AESABESP Congresso Nacional de Saneamento e Meio Ambiente, São Paulo, SP, Brasil, 27. Recuperado de http://rigeo.cprm.gov.br/jspui/bitstream/doc/16643/3/201608\%20-\%20Chuva\%20Mensal\%20e\%20Anual\%20em\%20S\%c3\%a3o\%20Paulo\%20Capital\%20-\%20Marcuzzo.pdf.

Medeiros, F.J., Lucio, P. S., \& Silva, H.J.F. (2017). Análise de Métodos de Krigagem na Estimativa da Precipitação no Estado do Rio Grande do Norte. Revista Brasileira de Geografia Física, 10(5), 1668-1676.

Mello, Y. R., \& Sampaio, T. V. M. (2019). Análise geoestatística da precipitação média para o estado do Paraná. Revista Brasileira de Climatologia, 25, 643660 .

Mendes, A. T., \& Zukowski Junior, C. C. (2019). Caracterização do Regime Pluviométrico do Município de Araguaína-TO. Revista Brasileira de Meteorologia, 34(4), 449-458.

Mendonça, M. C. S., Sena, I. M. N., \& Santos, M. R. A. (2015). Comparação dos métodos da média aritmética e de Thiessen para determinação da pluviosidade média da Sub-Bacia do Rio Siriri. Anais do Congresso Internacional da Rede de Saneamento e Abastecimento de Água (RESAG), Aracaju, SE, Brasil, 2. http://www.resag.org.br/congressoresag2015/anais/img/pdfs/ID_21.pdf.

Menezes, F. P. (2013). O uso da estatística para regionalização da precipitação no Estado do Pará. (Dissertação de Mestrado). Programa de Pós Graduação em Engenharia Civil, Universidade Federal do Pará (UFPA), Belém, PA, Brasil.

Mushtaha, A. M., Van Camp, M., \& Walraevens, K. (2019). Quantification of recharge and runoff from rainfall using new gis tool: example of the Gaza Strip aquifer. Water (Switzerland), 11(1).

Paiva, R. C. D. (2008). Estimativa de Campos de Precipitação: Análise Geoestatística e Integração de Dados de Postos Pluviométricos e Satélite TRMM.

Pereira, A. S., Shitsuka, D. M., Parreira, F. J., \& Shitsuka, R. (2018). Metodologia da pesquisa científica.

Ponte, J. P. X., Lima, J. J., Cardoso, A. C., \& Rodrigues, R. M., (2013). Análise do IBEU Local Região Metropolitana de Belém-PA. In L. C.de Q. Ribeiro, \& M. G. Ribeiro (Orgs.). Ibeu: índice de bem-estar urbano. Letra Capital.

Righi, E., \& Basso, L. A. (2016). Aplicação e análise de técnicas de interpolação para espacialização de chuvas. Ambiência Guarapuava (PR), 12 (1), 101-117.

Rossato, L., Alvalá, R. C., Marengo, J. A., Zeri, M., Cunha, A. P., Pires, L., \& Barbosa, H. A. (2017). Impact of soil moisture on crop yields over Brazilian semiarid. Frontiers in Environmental Science, 5.

Silva, C. S. D., Pereira, M. G., Delgado, R. C., \& Silva, E. V. D. (2016). Spatialization of soil chemical and physical attributes in an agroforestry system, Seropédica, Brazil. Cerne, 22(4), 407-414.

Silva, T. P., Rodrigues, R. A., Delgado, R. C., Oliveira, L. J. C., \& Sarmento, A. P. (2018). Métodos de interpolação espacial do regime pluviométrico no espaço urbano de Catalão, Goiás. Revista Brasileira de Geografia Física, 11(3), 745-757.

Silva, A. S., Anjos, M. M. S., Gomes, A. W. A., Bezerra, A. C., \& Guimarães, M. J. M. (2020). Modelagem automática e cálculo da precipitação média da Bacia Hidrográfica do Rio Mundaú. Research, Society and Development, 9(10), 1-24.

Shatha, I. J., \& Kadhum, J. H. (2019). Dynamical analysis of severe rain events over Iraq. Al-Mustansiriyah Journal of Science, 30(1).

Uliana, E. M., Reis, E. F., Silva, J. G. F., \& Xavier, A. C. (2013). Precipitação mensal e anual provável para o Estado do Espírito Santo. Irriga, $18(1)$, $139-417$.

Wanderley, H. S., Amorim, R. F. C. D., \& Carvalho, F. O. D. (2012). Variabilidade espacial e preenchimento de falhas de dados pluviométricos para o estado de Alagoas. Revista Brasileira de Meteorologia, 27(3), 347-354.

Wei, Z., Ruirui, L., Yan Quan, L., \& Jin, X. (2018). Effect of different areal precipitation estimation methods on the accuracy of a reservoir runoff inflow forecast model. Earth and Environmental Science, 208. 